Received: $\quad 2019.08 .27$ Accepted: 2019.10 .27 Published: 2020.01 .13

\title{
The Nephroprotective Effect of TNF Receptor- Associated Factor 6 (TRAF6) Blockade on LPS-Induced Acute Renal Injury Through the Inhibition if Inflammation and Oxidative Stress
}

Authors' Contribution: Study Design A Data Collection B Statistical Analysis C Data Interpretation D Manuscript Preparation E Literature Search F Funds Collection $G$
Corresponding Author: Source of support:

Background:

Material/Methods:

Results:

Conclusions:

MeSH Keywords:

Full-text PDF:
$A B C D$ Xuemei Chen*

BD 1 Yiqing Zhao*

AEFG 2 Jiajun $\mathrm{Xu}$

BD 1 Jiachun Bao

CE 1 Junyao Zhao

CD 1 Jingfeng Chen

EF 2 Guowei Chen

ACEFG 2 Jibo Han
1 Department of Pharmacy, Affiliated Hospital of Jiangnan University, Wuxi, Jiangsu, P.R. China

2 Department of Cardiology, The Second Affiliated Hospital of Jiaxing University, Jiaxing, Zhejiang, P.R. China

* Xuemei Chen and Yiqing Zhao contribute equally to this paper

Jibo Han, e-mail: jibohanjx2y@163.com

Financial support was provided by the Public Welfare Science and Technology Program of Jiaxing City (grant no. 2018AY32008, 2017AY33032; Jiaxing, China)

Inflammation and oxidative stress play important roles in the pathogenesis of acute kidney injury (AKI). TRAF6 functions as a signal transducer in the Toll-like receptor 4 signaling pathway. Several reports have previously implicated TRAF6 signaling in kidney pathology. Here, we investigated whether TRAF6 blockade can mitigate inflammatory responses and oxidative stress in AKI.

C57BL/6 mice were injected with lipopolysaccharide (LPS, $15 \mathrm{mg} / \mathrm{kg}$ ) to induce AKI. Double immunofluorescence staining of kidney tissues showed that TRAF6 was localized to renal tubular epithelial cells, and then a tubular epithelial cell line (NRK-52E) was used for in vitro analysis. TRAF6 was blocked in vitro using siRNA and in vivo using AAV2/2 shRNA.

The knockdown of TRAF6 in mice by AAV2-shTRAF6 significantly reduced renal inflammation, oxidative stress, apoptosis and kidney dysfunction in AKI. In vitro, silencing the expression of TRAF6 attenuated LPS $(0.5 \mu \mathrm{g} / \mathrm{mL})$-induced inflammatory responses and oxidative stress and upregulated proapoptotic factors. Furthermore, the beneficial actions of TRAF6 blockade were closely associated with its ability to increase IKB- $\alpha$ and Nrf2.

Our findings provide direct evidence that TRAF6 mediates LPS-induced inflammation and oxidative stress, leading to renal dysfunction. We also show that TRAF6 inhibition is a potential therapeutic option to prevent AKI.

Acute Kidney Injury • Inflammation • Lipopolysaccharides • Oxidative Stress • TNF Receptor-Associated Factor 6

https://www.medscimonit.com/abstract/index/idArt/919698

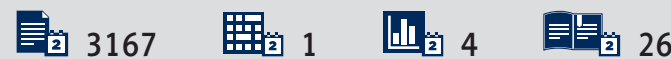




\section{Background}

Acute kidney injury (AKI), an abrupt loss of renal function, increases the risk of mortality during the development of an uncontrolled systemic inflammatory response [1]. AKI can result from various events, including sepsis, organ transplantation, cardiac surgery and rheumatic fever [2-4]. Among these, sepsis is consistently identified as the most important cause of AKI [1]. Extensive studies have revealed potential mediators, including a surge of oxidative stress, inflammation, apoptosis, increased serum creatinine levels and decreased urine output [5-9]. Among these various structural and functional events, inflammation and oxidative stress seem to be upstream of the cascade. Sepsis has been demonstrated to induce inflammation and oxidative stress in the kidneys, leading to cell apoptosis and renal dysfunction [5,7]. Despite the growing understanding of its pathophysiology, the treatment of septic AKI is still not satisfactory. Thus, exploring new and effective therapeutic options to prevent its spread is necessary.

Toll-like receptor 4 (TLR4) is a pattern-recognition receptor for lipopolysaccharide (LPS) [10]. TLR4 uses tumor necrosis factor receptor-associated factor 6 (TRAF6) as a bridging adaptor to recruit IRAK and TAK1 to activate the nuclear factor (NF)-KB pathway [11]. The translocation of NF- $\mathrm{KB}$ to the nucleus induces the expression of target genes, including tumor necrosis factor (TNF)- $\alpha$, interleukin 6 (IL-6), and other inflammatory cytokines.

TRAF6 is the only intracellular protein that transmits signals from the intracellular portion of IL-1R/TLR family members and TNFR superfamily members in different cell types [11]. Several reports have previously implicated TRAF6 signaling in kidney pathology. In primary rat proximal renal tubular cells, silencing TRAF6 by siRNA inhibits the LPS-induced inflammatory response and promotes cell survival [12]. In animal models, LPS-induced acute renal failure is associated with the upregulation of proinflammatory factors and activation of caspase3, while miR-590-3p attenuates LPS-induced AKI and podocyte apoptosis by targeting TRAF6 [13].

To the best of our knowledge, there have been no reports on TRAF6 knockdown in a sepsis-induced mouse model of AKI. In this study, we investigated the potential effect of TRAF6 knockdown against inflammatory responses and oxidative stress in AKI. Analysis of kidney tissues showed upregulated expression of TRAF6 in the renal tubular epithelial cells of LPS-induced AKI mice. Using an AKI mouse model, we show that knocking down TRAF6 with AAV2/2-shTRAF6 attenuated indices of inflammation and oxidative stress in kidneys and protected against renal dysfunction. The results obtained by silencing TRAF6 with siRNA in cultured renal tubular epithelial cells challenged with LPS mirrored the results obtained in our in vivo experiments.

\section{Material and Methods}

\section{Reagents}

LPS (from Salmonella typhosa) was purchased from Sigma (St. Louis, MO). Antibodies against TRAF6 (ab33915,1: 1000 dilution) and 3-nitrotyrosine (3-NT; ab61392,1: 300 dilution) were obtained from Abcam (Cambridge, USA). Antibodies against transforming growth factor- $\beta$ (TGF- $\beta$; sc-146, 1: 300 dilution), collagen (col) IV (sc-29010,1: 300 dilution), Bax (sc-7480,1: 300 dilution), Bcl-2 (sc-7382,1: 300 dilution), IKB- $\alpha($ sc-373893,1: 300 dilution), heme oxygenase-1 (HO-1; sc-136960,1: 300 dilution), GAPDH (sc-32233,1: 300 dilution) and the secondary horseradish peroxidase-conjugated antibody (7074, 1: 5000 dilution) were obtained from Santa Cruz Biotechnology. Antibodies against Nrf2 was obtained from Cell Signaling Technology (CST, CA, USA).

\section{Animal experiments}

Male C57BL/6 mice (18-22 g) were purchased from Wenzhou Medical University Animal Centre (Wenzhou, China). All mice received humane care in line accordance with the National Institutes of Health guidelines. All mice experimental procedures and care were approved by the Affiliated Hospital of Jiangnan University Animal Policy and Welfare Committee.

Adeno-associated virus type/mutant 2 (AAV2) was stable expressed in the kidney [14]. For TRAF6 knockdown, AAV2/2-U6shTRAF6 targeting TRAF6 (titer, $2.6 \times 10^{12} \mathrm{GC} / \mathrm{mL}$ ) and negative control AAV2/2-U6-NC (titer, $6.4 \times 10^{12} \mathrm{GC} / \mathrm{mL}$ ) from Genechem (Shanghai, China) were used. The following sequences were used: 5'-aattcgCAGGTATCTTGAGAAGCCAATGGAAttcaagagaTTCCATTGGCTTCTCAAGATACCTGttttttg-3' for shTRAF6 and 5'-aattcgTTCTCCGAACGTGTCACGTAAttcaagagaTTACGTGACACGTTCGGAGAAttttttg-3' for the negative control. We injected AAV2 expressing TRAF6 shRNA or negative control sequence into the tail vein one week before LPS injection (AAV2/2 shTRAF6treated group or AAV2/2 NC-treated group).

Mice were initially randomly divided into 2 groups ( $n=14$ per group). The animals in the AAV2/2 NC-treated group were further allocated into 2 groups: 7 mice were randomly chosen to receive an intraperitoneal injection of $15 \mathrm{mg} / \mathrm{kg}$ LPS while the other 7 mice received an equal volume of vehicle (phosphate buffered saline (PBS)) daily. Similarly, the AAV2/2 shTRAF6treated animals were randomly divided into groups and received an injection of either LPS $(n=7)$ or PBS $(n=7): 1)$ the AAV2/2 NCtreated control mice that received vehicle (NC); 2) the AAV2/2 NC-treated mice that received LPS (NC+LPS); 3) the AAV2/2 shTRAF6-treated mice that received LPS (shTRAF6+LPS); 4) the AAV2/2 shTRAF6-treated mice that received PBS (shTRAF6). Twenty-four hours following the initiation of LPS treatment, 
the animals were anesthetized by pentobarbital sodium (1\%, $40 \mathrm{mg} / \mathrm{kg}$, i.p.) and sacrificed. Blood and kidney were collected. Serum $\mathrm{Cr}$ and UN were determined using commercial kits.

\section{Histological assessments}

Kidney tissues were fixed in paraformaldehyde and embedded in paraffin, then cut into 5 - $\mu \mathrm{m}$ sections and stained with hematoxylin \& eosin for histology. The stained sections were observed using microscope (200x amplification).

Apoptosis was measured by TUNEL (RD). Images were observed under a fluorescence microscope (200x amplification).

For double labeling, sections were incubated with both a TRAF6 antibody and an antibody against aquaporin 1 (AQO-1, Santa Cruz Biotechnology; sc-32737, 1: 200 dilution) or Wilms' tumor 1 (WT-1, Novus Biologicals, Littleton, CO; NBP2-44607, 1: 200 dilution). The slides were then incubated with 2 secondary antibodies (TRITC-labeled, Abcam, ab6786, 1: 500 dilution or Alexa Fluor 488- labeled, Abcam, ab150077, 1: 500 dilution) for $1 \mathrm{~h}$ at room temperature.

Kidney tissue sections were deparaffinized and hydrated for immunohistochemistry for 3-NT [15].

\section{Determination of cytokine levels}

IL- 6 and TNF- $\alpha$ proteins in renal and serum were detected using cytokine-specific ELISA kits (eBiosciences Inc, CA, USA).

\section{Cell culture studies}

Rat tubular epithelial NRK-52E cells were purchased from the Shanghai Institute of Biochemistry and Cell Biology. NRK-52E cells were grown in DMEM containing glucose $(5.5 \mathrm{mM})$, $\mathrm{FBS}(5 \%)$, penicillin and streptomycin $(100 \mathrm{U} / \mathrm{mL})$.

TRAF6 were silenced in NRFK-52E cells through siRNA (5'-AGGAGACAGGUUUCUUGUGdTdT-3') using LipofectAMINE ${ }^{\text {TM }}$ 2000 (Invitrogen, Carlsbad, California). Knockdown was verified by Western blotting.

\section{Determination of superoxide (02-)}

Superoxide (02-) were determined using DHE (5 $\mu \mathrm{M})$ [15]. Images were observed under fluorescence microscope (200x amplification).

\section{Western blotting}

The common procedure for western blotting was described in our previous publication [16].Renal tissues and cells were lysed, and the protein concentration was determined by the Bradford assay. Lysates were separated by sodium dodecyl sulfate-polyacrylamide gel electrophoresis and electrotransferred to nitrocellulose membranes. The membranes were blocked for $1.5 \mathrm{~h}$ in Tris-buffered saline (pH 7.6) containing 0.05\% Tween 20 and 5\% nonfat dry milk at room temperature. Primary antibody incubations were carried out at $4{ }^{\circ} \mathrm{C}$ overnight. Secondary antibodies were applied for $1 \mathrm{~h}$ at room temperature. Immunoreactivity was visualized using enhanced chemiluminescence reagents (Bio-Rad Laboratories, Hercules, CA) and quantified using ImageJ analysis software version 1.38 e $(\mathrm{NIH}$, Bethesda, MD, USA). Values were normalized to respective protein controls.

\section{Real-time quantitative qPCR}

The common procedure for RT-qPCR was described in our previous publication [16]. Cells and renal tissues were homogenized in TRIZOL. RT-qPCR was carried out using a two-step M-MLV Platinum SYBR Green qPCR SuperMix-UDG kit. Primers for genes including TGF- $\beta$, Col-IV, Bax, Bcl-2, Nrf2, HO-1, TNF- $\alpha$, IL- 6 and $\beta$-actin were synthesized by Invitrogen. The primer sequences used are shown in Table 1. Target mRNA was normalized to $\beta$-actin.

\section{Statistical analysis}

All experiments involved randomization procedures and were conducted in a blinded manner. The in vitro experiments were repeated at least 3 times. The data are presented as the means \pm SEMs. The data were normally distributed, and we tested the distribution with the Kolmogorov-Smirnov test. The statistical significance of the differences between groups was determined by Student's t-test or ANOVA for multiple comparisons in GraphPad Pro 5.0 (GraphPad, San Diego, CA). We used one-way ANOVA followed by Dunnett's post hoc test when comparing data from more than two groups and oneway ANOVA nonparametric Kruskal-Wallis test followed by Dunn's post hoc test when comparing multiple independent groups. Differences were considered to be significant at $p<0.05$.

\section{Results}

TRAF6 expression is upregulated in renal tubular epithelial cells in LPS-induced AKI mice

Our first objective was to determine whether TRAF6 is involved in LPS-related AKI. We detected TRAF6 protein in kidney tissues of LPS-induced AKI mice. As shown in Figure 1A, the level of TRAF6 was increased compared to that in control mice. Double immunofluorescence staining showed TRAF6 immunoreactivity in AQP-1-positive tubular epithelial cells (Figure 1B). TRAF6 immunoreactivity, although significantly lower, was also observed 
Table 1. Sequences of primers for real-time qPCR assay used in the study.

\begin{tabular}{|c|c|c|c|}
\hline Gene & Species & FW & RW \\
\hline TGF- $\beta$ & Mouse & TGACGTCACTGGAGTTGTACGG & GGTTCATGTCATGGATGGTGC \\
\hline Col-IV & Mouse & GCTCCACCACTCAAAGGTGTT & GGCACAGTCGAGTCTTCCA \\
\hline Bax & Mouse & TGAAGACAGGGGCCTTTTTG & AATTCGCCGGAGACACTCG \\
\hline $\mathrm{Bcl}-2$ & Mouse & ATGCCTTTGTGGAACTATATGGC & GGTATGCACCCAGAGTGATGC \\
\hline Nrf2 & Mouse & TTTTCCATTCCCGAATTACAGT & AGGAGATCGATGAGTAAAAATGGT \\
\hline $\mathrm{HO}-1$ & Mouse & GAGCAGAACCAGCCTGAACT & TTTGAACTTGGTGGGGCTGT \\
\hline$\beta$-actin & Mouse & CCGTGAAAAGATGACCCAGA & TACGACCAGAGGCATACAG \\
\hline TNF- $\alpha$ & Rat & ACTCCCAGGTTCTCTTCAAGG & GGAGGCTGACTTTCTCCTGGTA \\
\hline IL-6 & Rat & GAGTTGTGCAATGGCAATTC & ACTCCAGAAGACCAGAGCAG \\
\hline Nrf2 & Rat & ACTGTCCCCAGCCCAGAGGC & CCAGGCGGTGGGTCTCCGTA \\
\hline $\mathrm{HO}-1$ & Rat & TCTATCGTGCTCGCATGAAC & CAGCTCCTCAAACAGCTCAA \\
\hline$\beta$-actin & Rat & AAGTCCCTCACCCTCCCAAAAG & AAGCAATGCTGTCACCTTCCC \\
\hline
\end{tabular}

in WT-1-positive podocytes and glomeruli cells (Figure 1C). These findings show that the role of TRAF6 in LPS-induced AKI and renal tubular epithelial cells may be the major contributor to the inflammatory response.

\section{Knocking down TRAF6 leads to the preservation of renal function in LPS mice and the normalization of tissue apoptosis}

To confirm the role of TRAF6 in AKI, we silenced TRAF6 using AAV2/2-shTRAF6. AAV2/2 has been reported to target kidney tissues for transgene expression [14]. We injected AAV2/2-shTRAF6 through the tail vein one week prior to LPS injection. We confirmed knockdown by Western blotting (Figure 2A). Importantly, we showed that the knockdown of TRAF6 normalized the LPSinduced increase in the levels of serum urea nitrogen (UN) and creatinine $(\mathrm{Cr}$ ) (Figure 2B, 2C, respectively). The knockdown of TRAF6 was able to normalize the morphological alterations in renal tubular dilation, distortion and tubular epithelial cell edema in LPS-treated mouse kidneys (Figure 2D). Furthermore, silencing TRAF6 reduced the mRNA levels of the profibrotic factors transforming growth factor- $\beta$ (TGF- $\beta$ ) and collagen IV in LPS-treated mouse kidneys (Figure 2E). These results show that knocking down TRAF6 protects against LPS-induced renal dysfunction.

To assess whether the effects of TRAF6 deficiency on preserving renal function in AKI are mediated by the modulation of cell apoptosis, we measured the proapoptotic proteins Bax and the antiapoptotic protein Bcl-2. RT-qPCR analysis showed increased levels of Bax and decreased levels of Bcl-2 (Figure 2F). TRAF6 knockdown significantly reversed the changes in these apoptosis-related genes induced by LPS. TUNEL staining of kidney specimens from LPS mice showed increased TUNEL-positive apoptotic cells (Figure 2G). However, treatment with TRAF6 deficiency prevented this increase. These findings strongly suggest that TRAF6 deficiency protects against LPS-related renal dysfunction and cell apoptosis in mouse kidneys.

\section{Knockdown of TRAF6 normalizes the LPS-induced release of inflammatory factor in the serum and kidney tissues}

We next assessed whether inflammation is involved in the actions of TRAF6 deficiency in AKI. We first measured serum TNF- $\alpha$ and IL6 levels by ELISA. These proinflammatory factors were increased in LPS-treated mice (Figure 3A, 3B, respectively). This increase was associated with increased levels of TNF- $\alpha$ and IL- 6 in kidney tissues (Figure 3C, 3D, respectively). TRAF6 knockdown reduced the release of TNF- $\alpha$ and IL- 6 in the serum and kidney tissues. The release of TNF and IL- 6 may be a humoral response secondary to LPS injection. We have performed an additional analysis to detect the infiltration of macrophages in the kidney tissues of AKI mice. We stained kidney tissues for CD68 and found increased immunoreactivity in AKI mice compared to NC mice (Figure 3E). CD68 immunoreactivity was reduced in kidney tissues of TRAF6-deficient AKI mice, suggesting decreased infiltration. These findings indicated that TRAF6 deficiency protects against LPS-related renal inflammation in AKI.

\section{TRAF6 deficiency prevents LPS- induced renal oxidative stress in vivo}

To analyze oxidative stress in renal tissues, we applied two assays. First, we stained renal tissues for an oxidative stress marker 3-NT. Next, we stained the sections for DHE. These measures were increased in kidney tissues of LPS-treated mice (Figure 3F, 3G, respectively). However, TRAF6 knockdown markedly attenuated LPS-induced renal oxidative stress. 
A

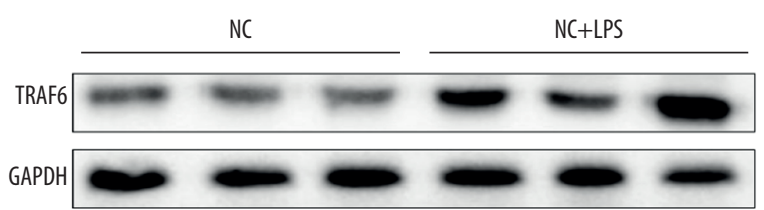

TRAF6/GAPDH

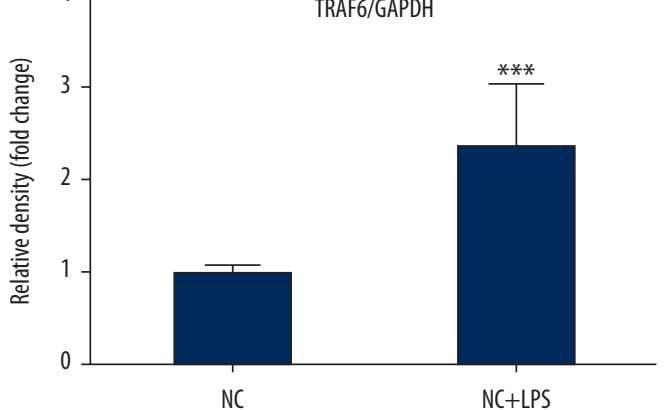

DAPI
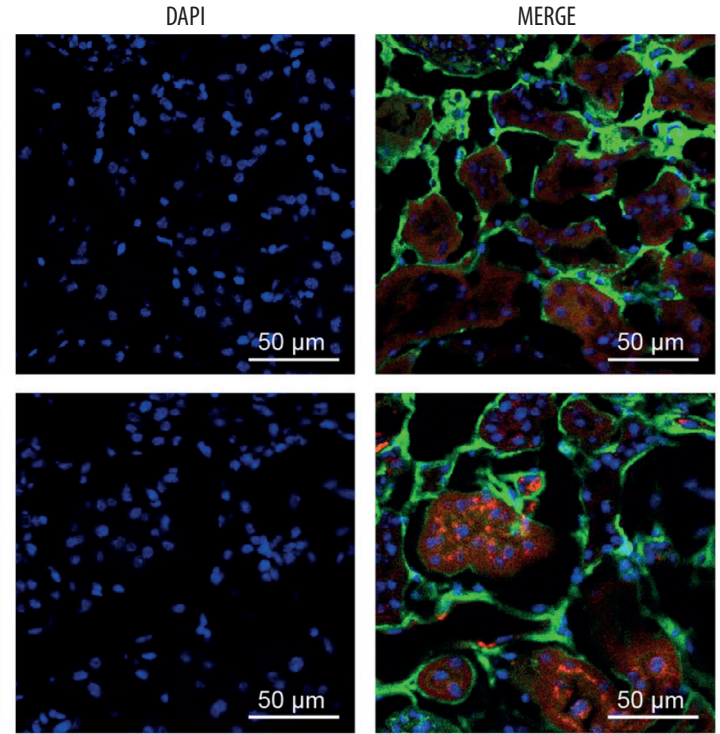

DAPI
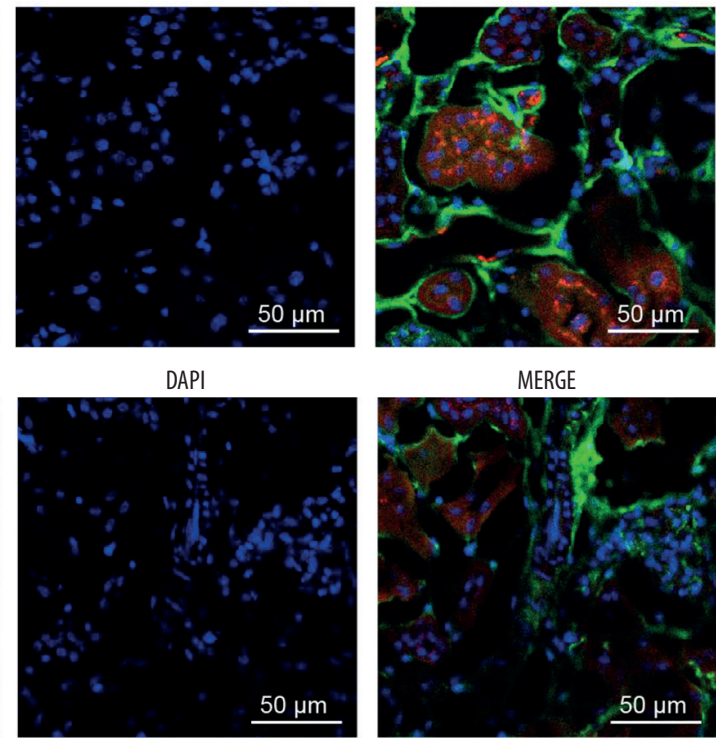

MERGE
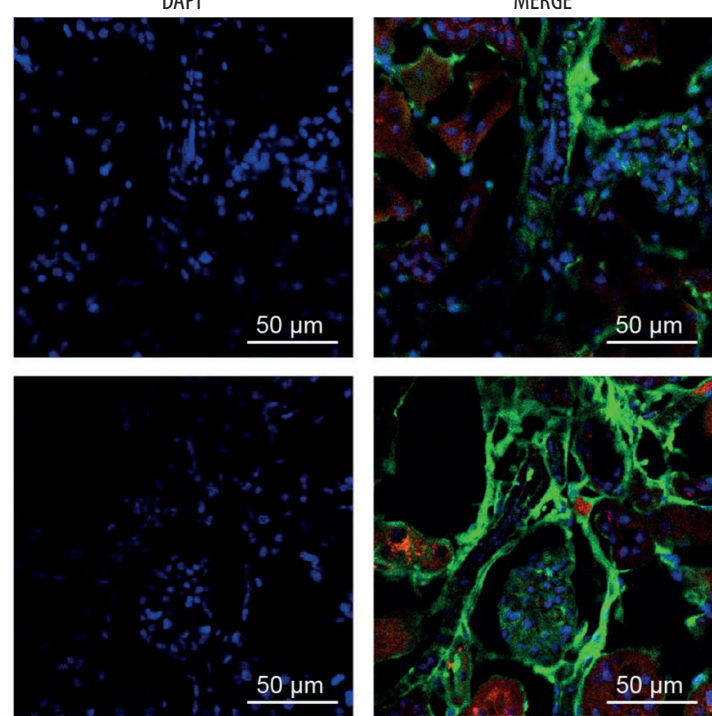

$50 \mu \mathrm{m}$

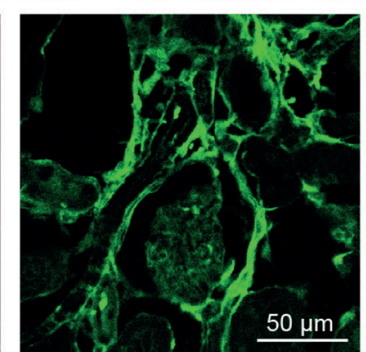

Figure 1. TRAF6 expression is upregulated in renal tissues in LPS mice. (A) Western blot analysis and densitometric analysis of TRAF6 levels in renal tissues. (B) Immunofluorescence staining for TRAF6 (red) and AQO-1 (green) showing immunoreactivity for TRAF6 in tubular epithelial cells [scale bar $=50 \mu \mathrm{m}$ ]. Merged images (yellow) showing colocalization. (C) Immunofluorescence staining of kidney tissues for TRAF6 (red) and WT-1 (green) [scale bar $=50 \mu \mathrm{m}$ ]. WT-1 was used as a marker of podocytes (NC=negative control; $n=7$ per group; ${ }^{*} v$ s. the NC group; ${ }^{* *} \mathrm{P}<0.001$ ). 


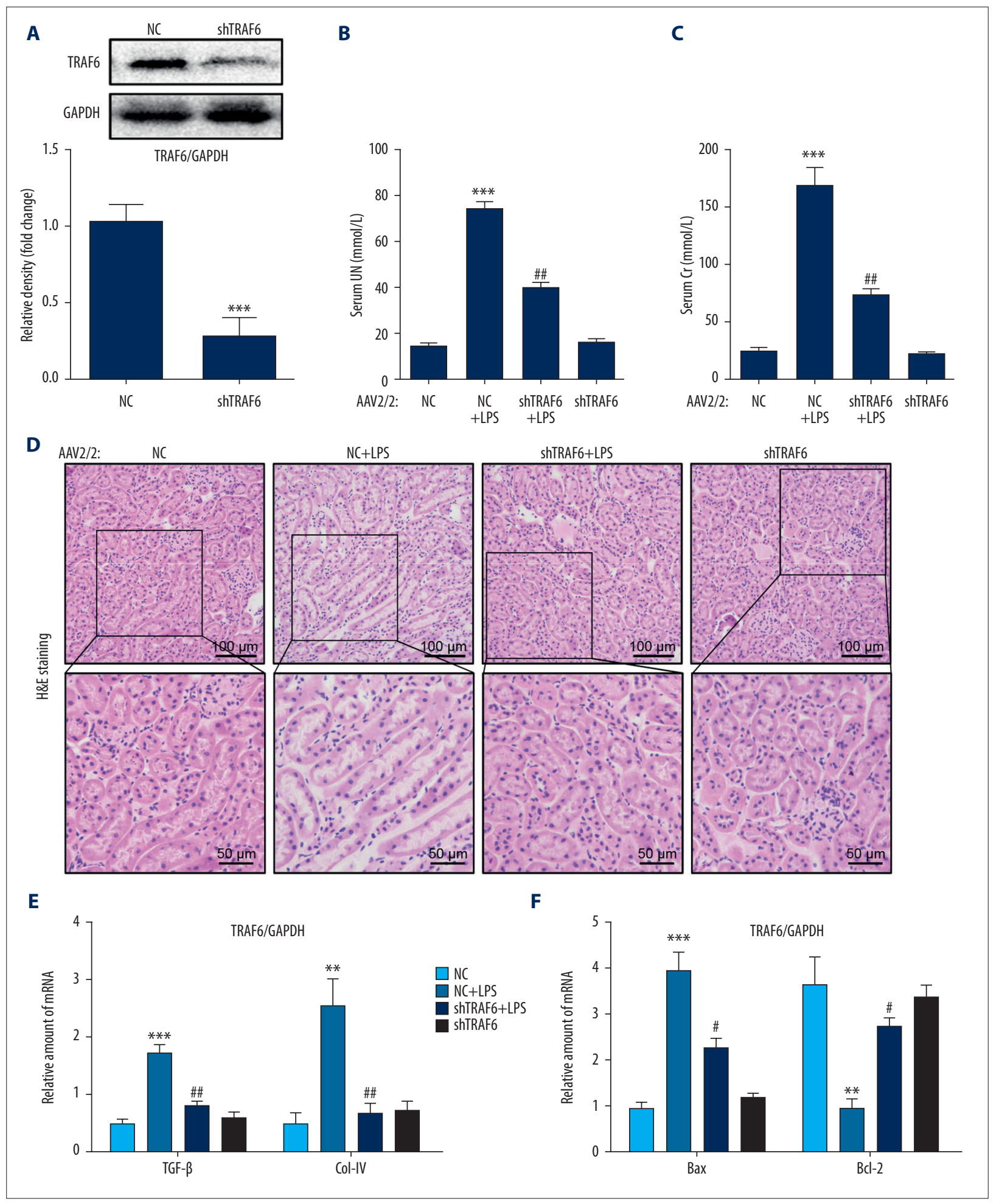




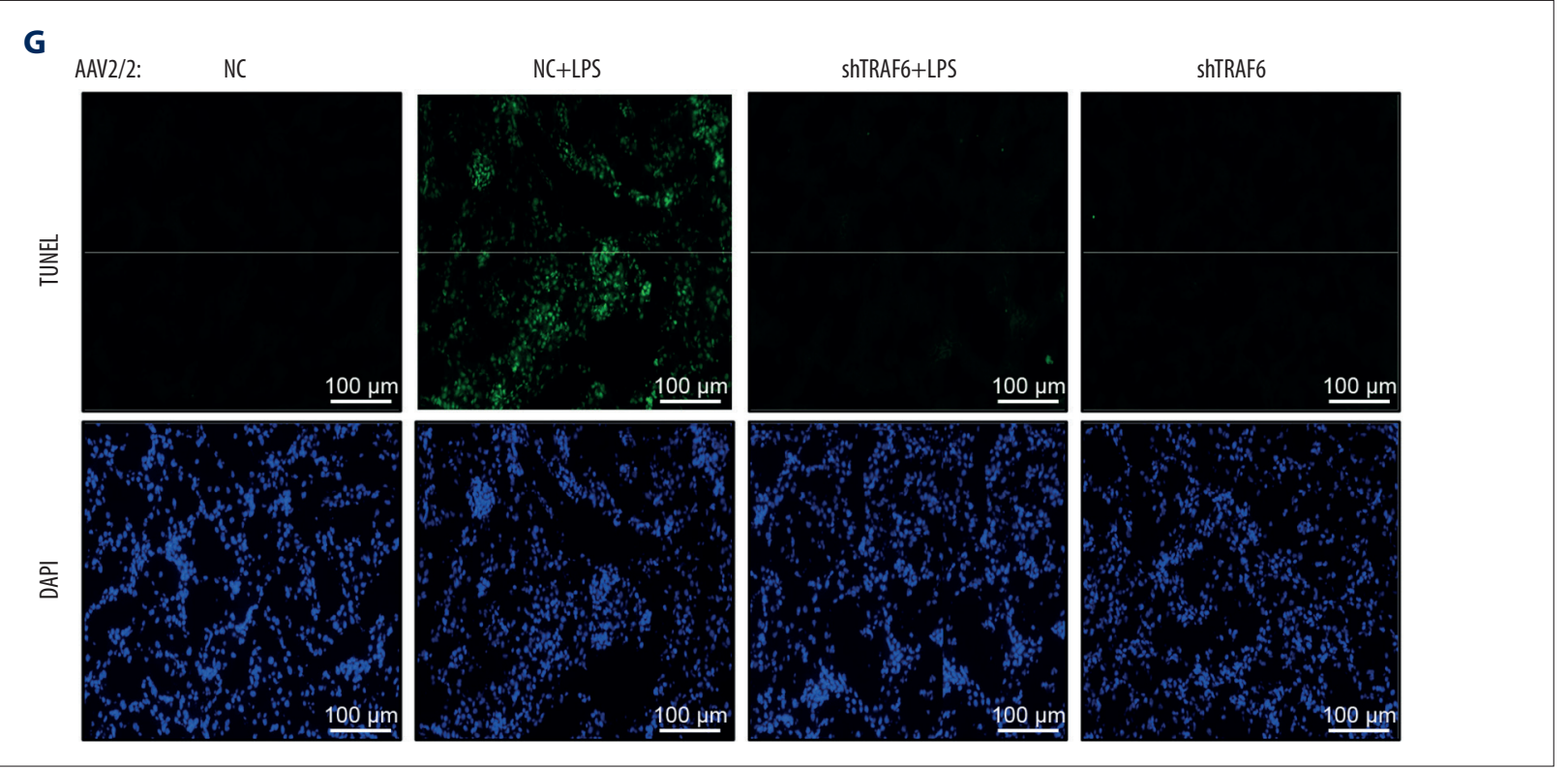

Figure 2. Knocking down TRAF6 leads to the preservation of renal function in LPS-treated mice and the normalization of tissue apoptosis. (A) The efficiency of TRAF6 knockdown following AAV2-shTRAF6 administration. (B, C) The levels of serum urea nitrogen (UN) and creatinine (Cr) in LPS-treated mice and mice treated with AZD. (D) Representative images of H\&E staining [scale bars $=100 \mu \mathrm{m}$ (upper) and $50 \mu \mathrm{m}$ (below)]. (E, F) The mRNA levels of TGF- $\beta$, Col-IV and Bax and Bcl- 2 in the kidney tissues of mice, as determined by qPCR [the mRNA levels were normalized to $\beta$-actin]. (G) Apoptotic DNA fragmentation in the renal tissues of LPS mice, as detected by TUNEL staining (green). The tissues were counterstained with DAPI (blue) [scale bar $=50 \mu \mathrm{m}$. ( $n=7$ per group; ${ }^{*}$ vs. the NC group; ${ }^{\#}$ vs. the NC+LPS group; ${ }^{\#} P<0.05,{ }^{* *}$ and $\left.{ }^{\# \# ~} P<0.01,{ }^{* * *} P<0.001\right)$.

To determine whether TRAF6 deficiency mediates its anti-oxidant effrct through the Nrf2 pathway, we detected Nrf2 and its downstream antioxidant gene HO-1. Our data showed that the expression of Nrf2 and HO-1 was induced when AKI mice were treated with AAV2-shTRAF6 (Figure $3 \mathrm{H}$ 3I, respectively). The NC mice treated with AAV2-shTRAF6 also exhibited increased levels of these antioxidant response genes; however, the data did not reach statistical significance.

\section{Silencing TRAF6 inhibits LPS-induced cell apoptosis and fibrosis in NRK-52E cells}

To confirm the role of TRAF6 in LPS-induced kidney dysfunction, we challenged cultured NRK-52E cells with LPS to model renal tubular epithelial cells in AKI. Transfecting NRK-52E cells with siRNA targeting TRAF6 (siTRAF6) led to decreased TRAF6 expression as expected (Figure 4A). Upon sustained LPS stimulation for $24 \mathrm{~h}$, the protein expression of Bax increased, and $\mathrm{Bcl}-2$ decreased (Figure 4B). Silencing TRAF6 reversed these changes. As shown in Figure 4C, LPS treatment induced the expression of the profibrotic factors TGF- $\beta$ and collagen IV, and the expression of both of these factors was reduced by siTRAF6. These findings suggest that siTRAF6 attenuates LPS-induced cell apoptosis and fibrosis in renal tubular epithelial cells.

\section{siTRAF6 attenuates LPS-induced inflammation and oxidative stress by increasing IKB- $\alpha$ and Nrf2}

To provide mechanistic insights into the role of TRAF6 in LPSassociated renal inflammation, we investigated the levels of proinflammatory factors in NRK-52E. siTRAF6 reduced LPSinduced TNF- $\alpha$ and IL- 6 mRNA levels (Figure 4D, 4E, respectively). We know from previous studies that the LPS-induced activation of NF- $\mathrm{KB}$ signaling causes renal inflammation $[17,18]$. We observed reduced activation of NF- $\kappa B$ by TRAF6 blockade, as evidenced by decreased degradation of IאB- $\alpha$ (Figure 4F) in LPS-stimulated NRK-52E cells.

Our animal experiments implicated inhibited oxidative stress as a potential mechanism by which TRAF6 blockade prevents LPS-induced renal deficits. We therefore measured antioxidant responses and ROS in NRK-52E cells exposed to LPS. Silencing TRAF6 caused an anti-oxidant effect in NRK-52E cells, as highlighted by increased protein expression (Figure 4G) and mRNA levels (Figure $4 \mathrm{H}, 4 \mathrm{I}$, respectively) of Nrf2 and $\mathrm{HO}-1$. The exposure of NRK-52E cells to LPS also increased intracellular ROS levels, as detected by DHE staining (Figure 4J). This ROS parameter was decreased when cells were transfected with siRNA targeting TRAF6. 


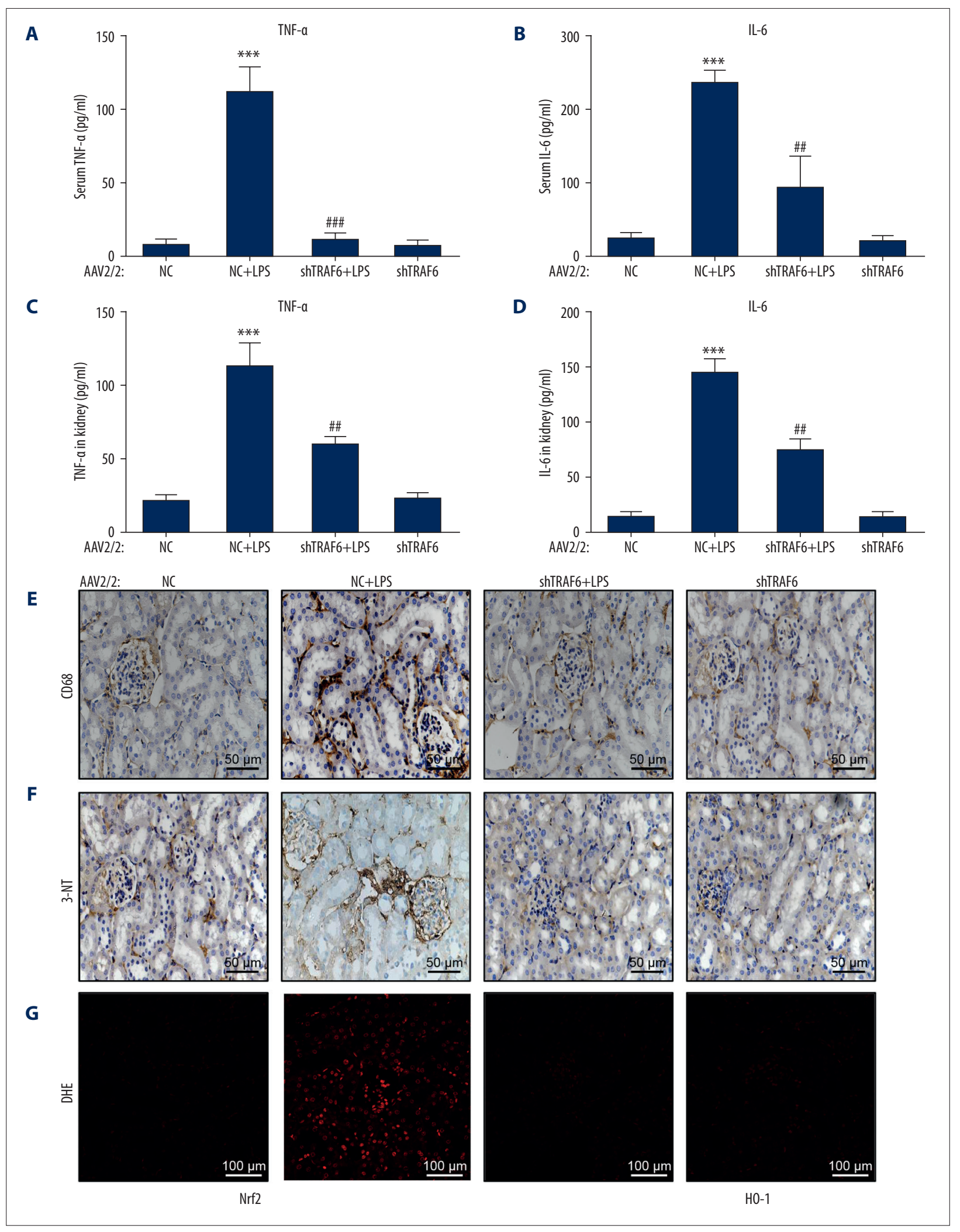




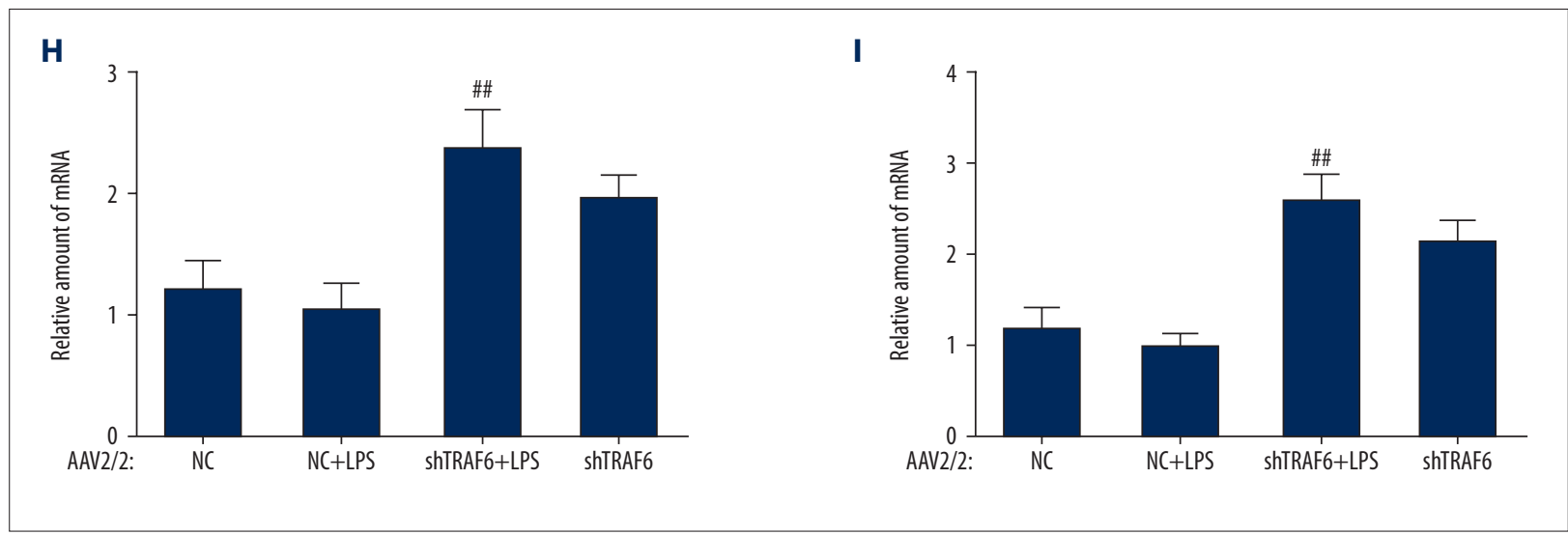

Figure 3. Knockdown of TRAF6 normalizes LPS-induced inflammatory factor release and oxidative stress in vivo. (A, B) The release of the inflammatory factors TNF- $\alpha$ and IL- 6 in the serum, as detected by ELISA. (C, D) The protein levels of TNF- $\alpha$ and IL- 6 in renal tissues, as detected by ELISA. (E) Representative immunohistochemcal staining of renal tissues for macrophage marker CD68. (E-G) LPS injection increased ROS production in the renal tissues of mice, as shown by increased 3-nitrotyrosine (3-NT; brown in E) chromogen staining and DHE (red in F) immunofluorescence. AAV2-shTRAF6 administration in mice prevented LPS-induced oxidative stress, as evidenced by reduced 3-NT staining and DHE fluorescence. $(\mathrm{H}, \mathrm{I})$ Antioxidant responses were measured in renal tissues by assessment the mRNA levels of Nrf2 and HO-1 ( $n=7$ per group; * vs. the NC group; ${ }^{*} v s$. the NC+LPS group; \#\# $\mathrm{P}<0.01$, ${ }^{* * *}$ and $\left.{ }^{\# \# ~} \mathrm{P}<0.001\right)$.

\section{Discussion}

The salient findings of our study include the demonstration that knocking down TRAF6 with AAV2/2-shTRAF6 protects against LPS-associated renal inflammation, oxidative stress, cell apoptosis, and functional deficits. Analysis of kidney tissues showed upregulated expression of TRAF6 in the renal tubular epithelial cells of LPS-induced AKI mice. The results obtained by silencing the protein expression of TRAF6 with siRNA in cultured renal tubular epithelial cells challenged with LPS mirrored the results obtained in our in vivo experiments. Furthermore, the beneficial actions of TRAF6 blockade are closely associated with its ability to increase IKB- $\alpha$ and Nrf2 (Figure 4K). Collectively, our findings provide evidence that TRAF6 is a potential therapeutic target for normalizing inflammation in acute renal injury.

AKI is a frequent complication of sepsis in critically ill patients with significant acute morbidity and mortality [1]. AKI represents a heterogeneous disease caused by various events, including sepsis, organ transplantation, cardiac surgery and rheumatic fever [2-4]. Among these, sepsis is consistently identified as the most important cause of AKI [1,19]. Recently, more attention has been focused on discovering new and effective therapeutic options for preventing or treating septic AKI. In the present study, we showed that knocking down TRAF6 with AAV2/2-shTRAF6 reduced LPS-induced cell apoptosis and renal dysfunction in vivo (Figure 2). TRAF6 expression has been reported in rat primary renal proximal tubule cells [12] and mouse podocytes [13]. We found that TRAF6 was upregulated in renal tubular epithelial cells in septic mice (Figure 1). Although studies are needed to examine the contribution of TRAF6 in other renal cells, we showed that the suppression of TRAF6 by siRNA in cultured renal tubular epithelial cells reduced molecular markers of fibrosis and apoptosis (Figure 4A-4C). These findings suggest that TRAF6 inhibition is protective against AKI and that TRAF6 plays an important role in the structural and functional characteristics of AKI.

Recent studies have shown that elevated and sustained inflammation, including increased levels of the proinflammatory cytokines IL- 6 and TNF- $\alpha$, is intricately linked to renal injuries [6]. It is believed that increased IL- 6 and TNF- $\alpha$ play a role in increased permeability, glomerular filtration rate, hemodynamic changes and renal fibrosis $[6,20,21]$. In addition, inflammatory cytokines may also cause cell apoptosis by inducing the expression of pro-apoptotic factors such as Bax [5]. In the present study, we showed that the suppression of TRAF6 by either AAV2/2-shTRAF6 (Figure 3A-3D) or siRNA (Figure 4D, 4E) reduced LPS-induced inflammation in vivo and in vitro. The overactivation of TRAF6/NF-KB inflammatory signaling leads to inflammation in the kidneys and the progression of AKI $[5,12,13,17,18]$. The activation of NF-KB results in increased pro-inflammatory cytokine production in LPS-induced renal damage $[17,18]$. Studies showing the inhibition of inflammation upon TRAF6 deficiency have further implicated this pathway in AKI [13]. Further implicating this pathway in AKI are study which show inhibition of inflammation upon TRAF6 deficiency [12]. In the TLR4 pathway, TRAF6 serves as a signal transducer that activates IKB kinase (IKK) and then IKB- $\alpha$ in response to proinflammatory mediators such as LPS. Based on these data, we hypothesized that TRAF6 blockade modulates inflammation by increasing $\mid \kappa B-\alpha$. Indeed, our 
results show that silencing TRAF6 reduces the activation of $N F-\kappa B$, as evidenced by the decreased degradation of $I \kappa B-\alpha$ (Figure 4F). Studies have also shown inhibition of TLR4 has also been shown to suppress LPS induced AKI [22]. What has remained elusive, however, is how TRAF6 is activated by LPS or other factors in AKI.
Oxidative stress has been linked to both the onset of sepsis as well as septic complications [23]. Recent studies have also shown that oxidative damage induced by reactive oxygen species (ROS) from LPS plays a critical role in septic injury in multiple organs, including the kidneys. The inhibition of ROS has been proven to have a protective effect against AKI $[7,24]$. In our study, we found that TRAF6 knockdown by AAV2/2-shTRAF6
A

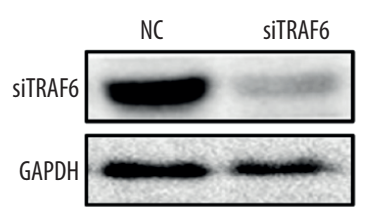

B

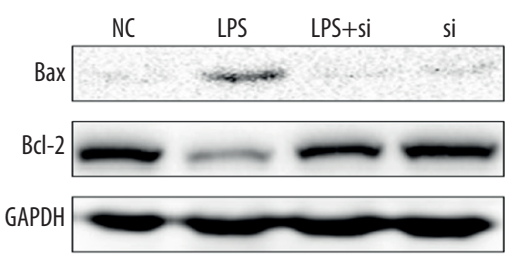

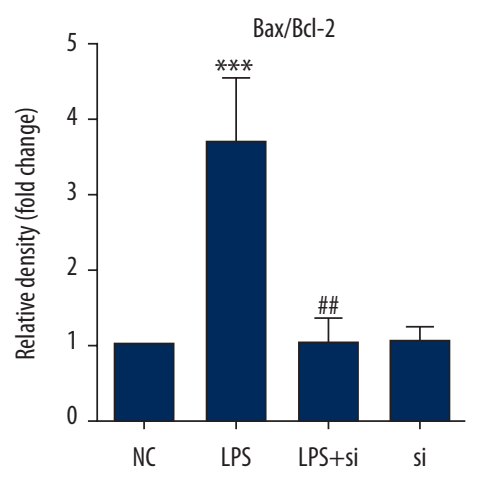

C

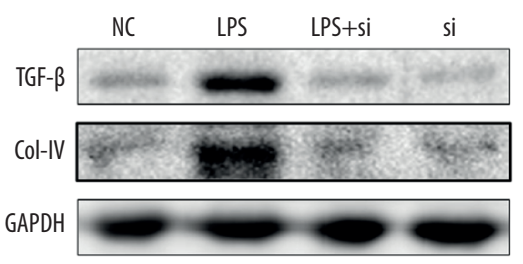

D

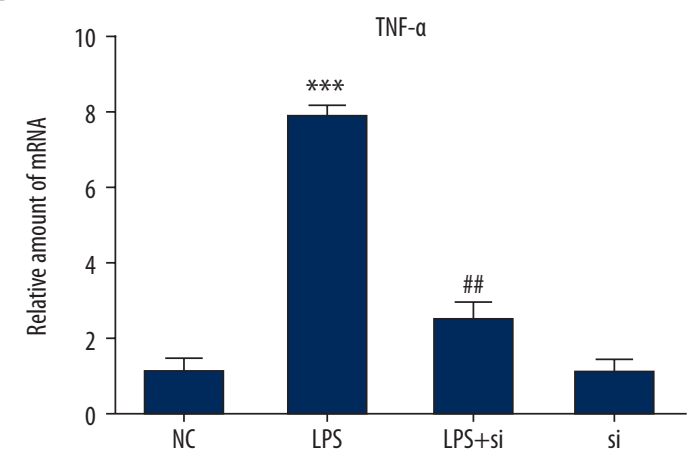

$\mathbf{F}$

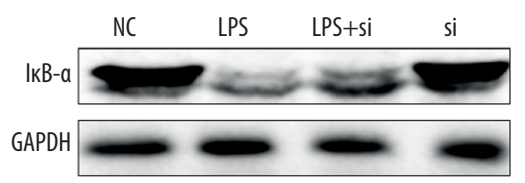

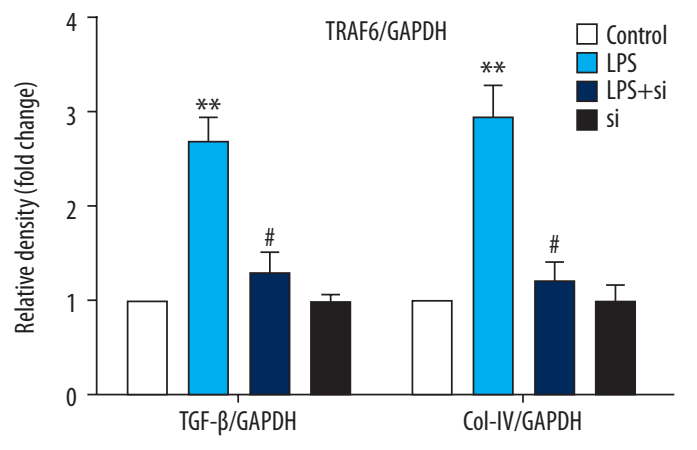

$\mathbf{E}$

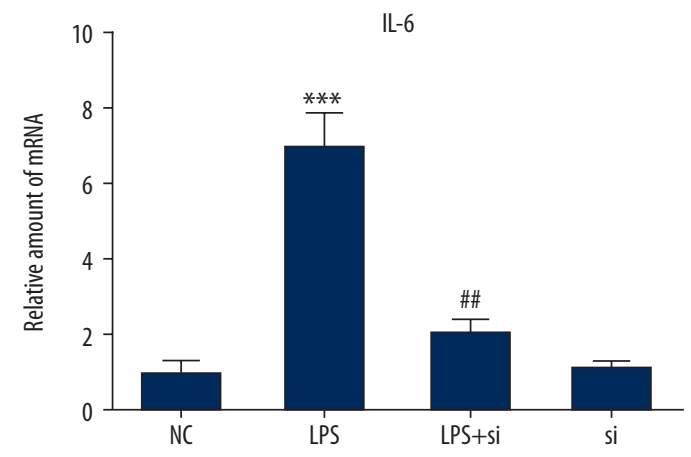

$\mathbf{G}$

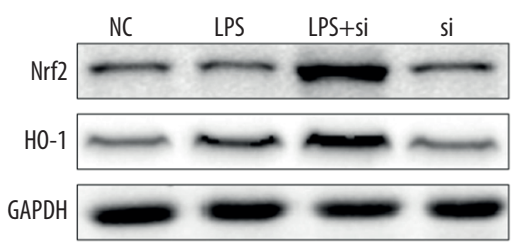

This work is licensed under Creative Common AttributionNonCommercial-NoDerivatives 4.0 International (CC BY-NC-ND 4.0)
Indexed in: [Current Contents/Clinical Medicine] [SCI Expanded] [ISI Alerting System] [ISI Journals Master List] [Index Medicus/MEDLINE] [EMBASE/Excerpta Medica] [Chemical Abstracts/CAS] 


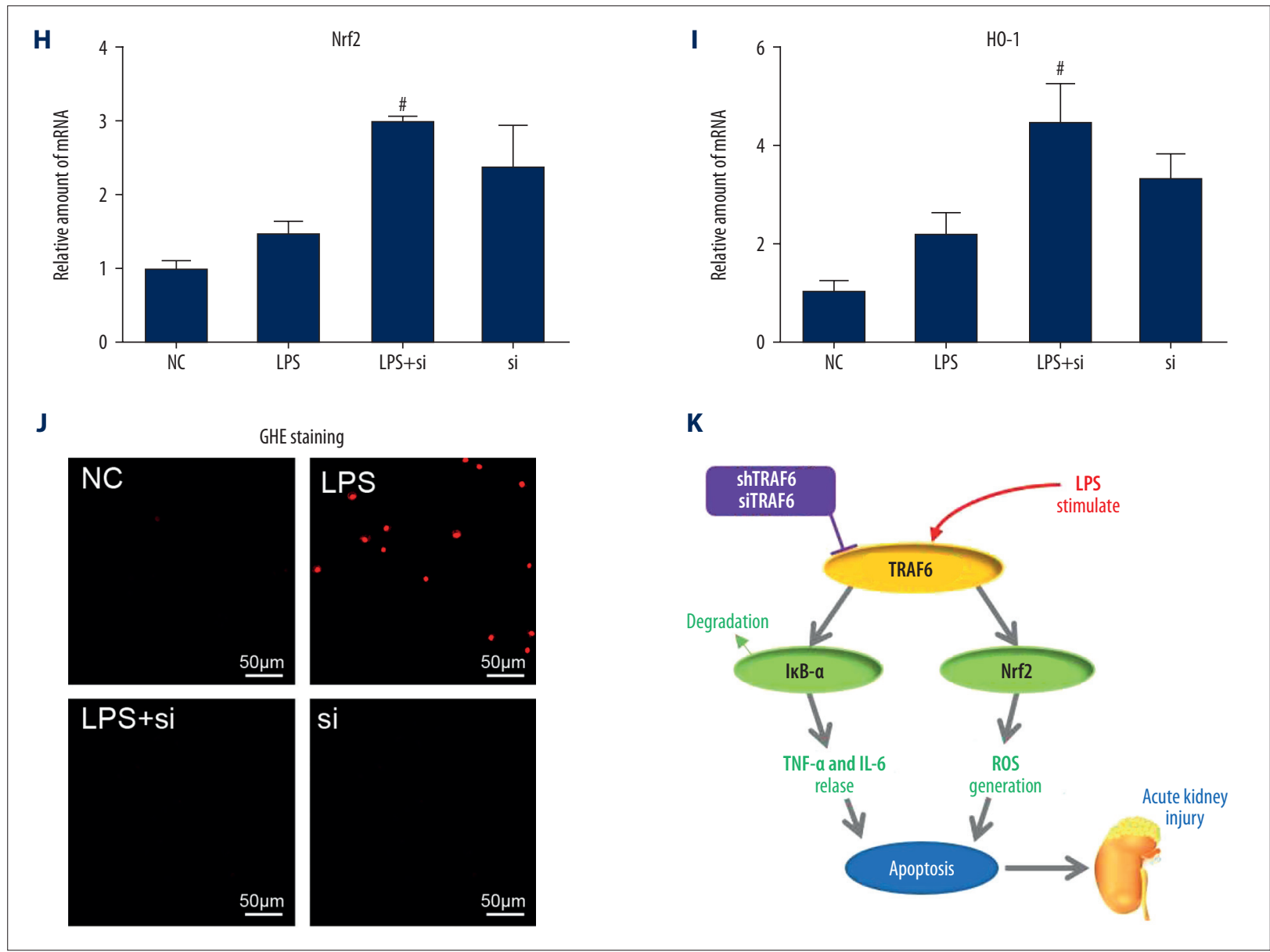

Figure 4. Silencing TRAF6 inhibits LPS-induced cell apoptosis, inflammation and oxidative stress by increasing IKB- $\alpha$ and Nrf2.

(A) NRK-52E cells were transfected with siRNA targeting TRAF6. Cells lysates were analyzed by Western blotting to measure TRAF6 levels. [NC=negative control; si=siTRAF6]. TRAF6-silenced NRK-52E cells were exposed to $0.5 \mu \mathrm{g} / \mathrm{mL}$ LPS for the indicated times. (B) After incubation with LPS for $24 \mathrm{~h}$, the expression of Bax and Bcl-2 in cell lysates was detected by immunoblotting. (C) After exposure to LPS for $24 \mathrm{~h}$, the protein levels of TGF- $\beta$ and Col-IV were detected by Western blotting. (D, E) After incubation with LPS for $6 \mathrm{~h}$, the mRNA levels of the inflammatory cytokines TNF- $\alpha$ and IL- 6 were detected by RT-qPCR. (F) After exposure to LPS for $1 \mathrm{~h}$, Western blot analysis of I $\mathrm{kb}-\alpha$ levels in NRK-52E cells was performed. (G) After exposure to LPS for $8 \mathrm{~h}$, siTRAF6 induced an antioxidant response in NRK-52E cells, as evidenced by increased protein levels of Nrf2 and HO-1. (H, I) After incubation with LPS for $6 \mathrm{~h}$, the mRNA expression of Nrf2 and HO-1 was measured in NRK-52E cells. (J) After exposure to LPS for $12 \mathrm{~h}$, ROS generation in NRK-52E cells was determined by dihydroethidium (DHE). (K) A schematic illustration of the prevention of LPS-induced injury in the kidneys by TRAF6 blockade ( ${ }^{*}$ vs. the NC group; \# vs. the LPS group; ${ }^{\#} \mathrm{P}<0.05$, ${ }^{* *}$ and ${ }^{\# \#} \mathrm{P}<0.01$, ${ }^{* * *}$ and ${ }^{\# \# \# ~} \mathrm{P}<0.001$.

protected against LPS-induced oxidative stress (Figure 3E, 3F). In vitro, silencing TRAF6 significantly attenuated LPS-induced ROS generation in NRK-52E cells (Figure 4J). Notably, Nrf2 signaling was activated by oxidative stress, suggesting that Nrf2 is essential for antioxidant activity [25]. Moreover, Nrf2-driven antioxidant response genes, such as $\mathrm{HO}-1$, are also known as effective phase II detoxifying enzymes [23]. We found that TRAF6 blockade induced the upregulation of $\mathrm{Nrf2}$ and the Nrf2-driven antioxidant response gene $\mathrm{HO}-1$ in vivo (Figure $3 \mathrm{G}, 3 \mathrm{H}$ ) and in vitro (Figure $4 \mathrm{G}-4 \mathrm{I})$. Although these are promising findings, the mechanism of Nrf2 induction by TRAF6 blockade is unclear. The currently accepted mechanism of Nrf2 regulation appears to primarily involve posttranslational mechanisms and the modulation of a binding protein called Kelch-like Erythroidcell-derived protein with CNC homology (ECH)-Associated Protein 1 (Keap 1) [26]. Determining whether TRAF6 blockade alters the keap1-Nrf2 interaction may open exciting possibilities for the treatment of AKI.

\section{Conclusions}

Our findings demonstrate that TRAF6 blockade attenuates LPSassociated renal inflammation, oxidative stress, apoptosis and 
renal dysfunction. Our results reveal that TRAF6 is primarily localized to renal tubular epithelial cells and that the beneficial actions of TRAF6 blockade are closely associated with its ability to increase I $\mathrm{KB}-\alpha$ and Nrf2. Collectively, our studies provide evidence that TRAF6 plays a significant role in inflammatory responses and oxidative stress in acute renal damage. Both methods of blocking TRAF6 may represent interesting therapeutic options to combat AKI.

\section{References:}

1. Dellepiane S, Marengo M, Cantaluppi V: Detrimental cross-talk between sepsis and acute kidney injury: New pathogenic mechanisms, early biomarkers and targeted therapies. Crit Care, 2016; 20: 61

2. Plataki M, Kashani K, Cabellogarza J at el: Predictors of acute kidney injury in septic shock patients: an observational cohort study. Clin J Am Soc Nephrol, 2011; 6: 1744-51

3. Thiele RH, Isbell JM, RosnerMH: AKI associated with cardiac surgery. Clin J Am Soc Nephrol, (2015; 10: 500-14

4. Li PKT, Burdmann EA, Mehta RL: Acute kidney injury: Global health alert. Transplantation, 2013; 95: 653-57

5. Woods JS, Dieguez-Acuna FJ, Ellis ME et al: Attenuation of nuclear factor kappa B (NF-kappaB) promotes apoptosis of kidney epithelial cells: A potential mechanism of mercury-induced nephrotoxicity. Environ Health Perspect, 2002; 110(Suppl.5): 819-22

6. Akcay A, Nguyen Q, Edelstein CL: Mediators of inflammation in acute kidney injury. Mediators Inflamm, 2009; 2009: 137072

7. Liu X, Lu J, Liao Y at el: Dihydroartemisinin attenuates lipopolysaccharideinduced acute kidney injury by inhibiting inflammation and oxidative stress. Biomed Pharmacother, 2019; 117: 109070

8. Javedan G, Shidfar F, Davoodi SH at el: Conjugated linoleic acid rat pretreatment reduces renal damage in ischemia/reperfusion injury: Unraveling antiapoptotic mechanisms and regulation of phosphorylated mammalian target of rapamycin. Mol Nutr Food Res, 2016; 60: 2665-77

9. Amani H, Habibey R, Shokri F at el: Selenium nanoparticles for targeted stroke therapy through modulation of inflammatory and metabolic signaling. Sci Rep, 2019; 9: 6044

10. Lucas $K$, Maes M: Role of the toll Like receptor (TLR) radical cycle in chronic inflammation: Possible treatments targeting the TLR4 pathway, Mol Neurobiol, 2013; 48: 190-204

11. Walsh MC, Lee J, Choi Y: Tumor necrosis factor receptor- associated factor 6 (TRAF6) regulation of development, function, and homeostasis of the immune system. Immunol Rev, 2015; 266: 72-92

12. Liu S, Lutz J, Chang J et al: TRAF6 knockdown promotes survival and inhibits inflammatory response to lipopolysaccharides in rat primary renal proximal tubule cells. Acta Physiol (Oxf), 2010; 199: 339-46

13. Ma J, Li YT, Zhang SX et al: MiR-590-3p attenuates acute kidney injury by inhibiting tumor necrosis factor receptor-associated factor 6 in septic mice. Inflammation, 2019; 42: 637-49

\section{Data availability}

All other data are included within the article or available from the authors on request.

\section{Conflict of interests}

None.

14. Qi YF, Li QH, Shenoy V at el: Comparison of the transduction efficiency of tyrosine-mutant adeno-associated virus serotype vectors in kidney. Clin Exp Pharmacol Physiol, 2013; 40: 53-55

15. Han J, Shi X, Zheng Z at el: Schisandrin B protects against angiotensin IIinduced endotheliocyte deficits by targeting Keap 1 and activating Nrf2 pathway. Drug Des Devel Ther, 2018; 12: 3985-97

16. Han J, Ye S, Zou C at el: Angiotensin II causes biphasic STAT3 activation through TLR4 to initiate cardiac remodeling. Hypertension, 2018; 72: 1301-11

17. Amoah-Apraku B, Chandler LJ, Harrison JK et al: NF-kappa B and transcriptional control of renal epithelial-inducible nitric oxide synthase. Kidney Int, 1995; 48: 674-82

18. Wang Y, Rangan GK, Goodwin B et al: Lipopolysaccharide-induced MCP-1 gene expression in rat tubular epithelial cells is nuclear factor-kappaB dependent. Kidney Int, 2000; 57: 2011-22

19. Harty J: Prevention and management of acute kidney injury. Ulster Med J, 2014; 83: 149-57

20. Furuichi K, Kaneko S, Wada T: Chemokine/chemokine receptor-mediated inflammation regulates pathologic changes from acute kidney injury to chronic kidney disease. Clin Exp Nephrol, (2009; 13: 9-14

21. Gomez H, Ince C, De Backer D at el: A unified theory of sepsis-induced acute kidney injury: Inflammation, microcirculatory dysfunction, bioenergetics, and the tubular cell adaptation to injury, Shock, 2014; 41: 3-11

22. Chen JL, John R, Richardson JA at el: Toll-like receptor 4 regulates early endothelial activation during ischemic acute kidney injury. Kidney Int, 2011; 79: 288-99

23. Koyner JL, Ali RS, Murray PT: Antioxidants. Do they have a place in the prevention or therapy of acute kidney injury? Nephron Exp Nephrol, 2008; 109: E109-17

24. Zhang $\mathrm{H}$, Chen MK, Li K et al: Eupafolin nanoparticle improves acute renal injury induced by LPS through inhibiting ROS and inflammation. Biomed Pharmacother, 2017; 85: 704-11

25. Kim J, Cha Y-N, Surh Y-J: A protective role of nuclear factor-erythroid 2-related factor-2 (Nrf2) in inflammatory disorders. Mutat Res, 2010; 690: 12-23

26. Marcotte D, Zeng W, Hus JC at el: Small molecules inhibit the interaction of Nrf2 and the Keap1 Kelch domain through a non-covalent mechanism. Bioorg Med Chem, 2013; 21: 4011-19 